\title{
Unveiling the Galaxy with Gaia
}

\author{
Antonella Vallenari \\ INAF, Padova Observatory \\ email: antonella.vallenari@oapd.inaf.it
}

\begin{abstract}
The Gaia Mission due for launch this year will revolutionise our understanding of the formation and evolution of the Milky Way and of the astrophysics in general. This paper reviews the current status of the Mission, and the expected accuracies as derived from instrument performance assessment.
\end{abstract}

Keywords. Astrometry, Galaxy:stellar content, Galaxy: structure

\section{Introduction}

The ESA Gaia mission is arguably the most exciting of the European flagship space missions of the coming decade. It will map the Galaxy down to $G=20$ mag, providing distances (parallaxes), space motions (proper motions and radial velocities) and astrophysical characterization (through time resolved multi-wavelength photometry and spectroscopy) for more than one billion stars throughout most of the Galaxy. In addition, several millions of galaxies and quasars will be observed. This huge and formidable database will allow us to address many key issues of Galactic astronomy, astrophysics and fundamental physics (for detailed overview of the science expectations, see among others Wilkinson et al. 2005, Turon et al. 2011). For example, it will revolutionize our knowledge of the structure and dynamics of our own Galaxy allowing the knowledge of its gravitational potential and mass distribution, including the dark matter component and the non-axisymmetric features such as spiral arms. The kinematic information will help to clarify the role of hierarchical formation and secular evolution in the shaping of the disks and the halo. This in turn will have obvious implications on our understanding of galaxy formation in general. It will lead to a remarkable improvement on the overall distance ladder, allowing the accurate and direct re-calibration of all of the secondary standard candles (see among others Turon et al. 2012, and Dopita et al. 2012). In addition, Gaia will provide highly accurate magnitudes and colors which will improve our knowledge of the stellar astrophysics, and of stellar populations in general, including open and globular clusters. Gaia will be sensitive to Jupiter-size planets with an orbital period of 1.5-9 years within 200 pc from us, giving a fundamental contribution to the planet science, in synergy with other techniques for planet detection and characterization (Sozzetti 2013). Concerning the solar system objects, Gaia will determine the orbits of hundreds of thousands of main-belt asteroids, near-Earth and Kuiper-Belt objects (Tanga \& Mignard 2012). Gaia will be complemented by on-going or proposed large-scale photometric and spectroscopic surveys, both in the visible and in the infrared: VPHAS+ (Drew 2012, UKIDSS, VVV VISTA (Saito et al. 2012) RAVE (Siebert 2012)), APOGEE (Majewski 2010), Hermes (Freeman 2012), Gaia-ESO Survey (Gilmore et al. 2012) and LAMOST Surveys as discussed in this Conference. 


\section{Gaia payload and telescopes}

A summary of the Gaia instruments, properties and expected performance can be found in de Bruijne (2012). Here we summarize some essential features. The Gaia spacecraft is built by EADS Astrium France and is scheduled for launch at the European Space port in French Guiana by a Soyuz-Fregat launcher in the second half of the year 2013. The Gaia Payload Module consists of two telescopes with a focal length of $35 \mathrm{~m}$, focusing the stars on a common focal plane by means of a beam combiner. The two M1 telescope mirrors $(1.45 \mathrm{~m} \times 0.5 \mathrm{~m})$ are fixed on the torus at a mutual angle of $106.5^{\circ}$, named the Basic Angle. The stability of this angle and in general of the whole structure is of fundamental importance to reach high astrometric accuracy. For this reason, the structure and the mirrors are made of Silicon Carbide, which ensures dimensional stability. However, small thermal gradient variations occurring within the payload can produce a small line of sight fluctuation which needs to be compensated. This is done by means of a continuous monitoring of the Basic Angle (BAM) using a Young-type interferometer to derive the requested corrections. The BAM measures the relative position of two interferometric patterns generated by a common laser diode source and focused on the same CCD in the focal plane of the telescope mirrors. Differential variations of the line-of-sight of each telescope are expected to produce differential fringe motion from which the required corrections can be estimated.

\subsection{The focal plane and the Gaia instruments}

To date, the Gaia focal plane is the largest sent into space (de Bruijne, Kohley, \& Prusti, 2010) being formed by 106 CCDs (see Fig. 1).

Each CCD consists of $4500 \times 1966$ pixels of physical dimension $10 \times 30 \mu m$ (for further detail see Short et al. 2013). As the satellite rotates, the sources move across the focal plane. The CCDs are read out synchronously with the source motion i.e in "time-delayed integration" (TDI). The integration time per CCD is about $4.4 \mathrm{sec}$, corresponding to 4500 TDI lines along scan. On the focal plane, the wave-front sensor and basic-angle monitor cover 2 CCDs each. Fourteen CCDs are used for the sky mapper (SM), where incoming images are first detected. This is the only part of the focal plane having separate

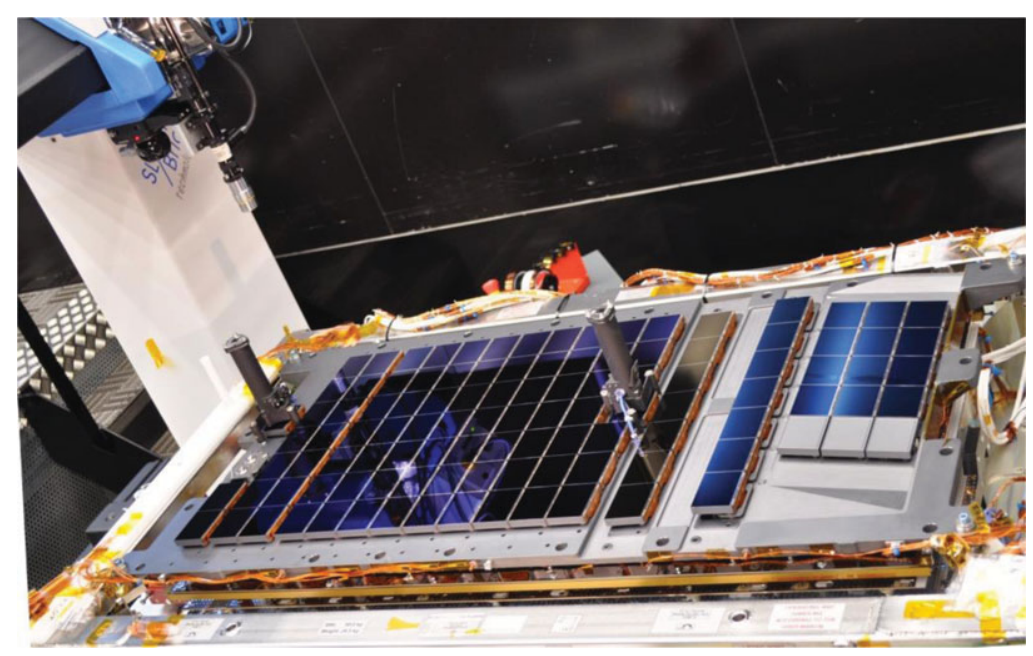

Figure 1. Gaia focal plane. Figure courtesy of of EADS-Astrium and ESA. 
CCDs for the two telescopes, while the rest of the focal plan is in common. The source detection is near-diffraction limited to about $0.1^{\prime \prime}$. The on-board detection and selection algorithms select continuously, in real-time, point sources brighter than $G=20$ and fainter than about $G=5.4 \mathrm{mag}$. The main astrometric field (AF), allocating sixty-two CCDs, is intended for angular-position measurements and records white light G-band (over $330-1050 \mathrm{~nm}$ ). The light is then dispersed by two prisms to produce the blue and red photometers (BP and RP) using 7 CCDs each and covering the wavelength range from 330 to $680 \mathrm{~nm}$, and from 640 to $1050 \mathrm{~nm}$, respectively. Both instruments produce low resolution spectra from which an integrated magnitude will be computed. Finally, at the trailing edge, the radial-velocity spectrometer (RVS) makes use of twelve CCDs. The RVS is a near-infrared instrument with a resolution of 11,200, that spans the wavelength range from 847 to $871 \mathrm{~nm}$. This range covers the Calcium triplet. Data from the RVS will be available for stars brighter than $\mathrm{V} \sim 16-16.5$ mag.

\subsection{The scanning law}

The satellite will operate at a distance of 1.5 million kilometres from Earth at the second Lagrange (L2) point of the Sun-Earth/Moon system, in the anti-Sun direction. Gaia will be inserted into a Lissajous-type orbit around L2, with an orbital period of 180 days, slowly scanning the sky while rotating. The Gaia nominal scanning law prescribes the attitude of the spacecraft during the operational phase determining when certain areas of the sky are observed. The scanning law is designed to fulfill several requirements such as providing a sky-sampling as uniform as possible. This implies a revolving scan law with constant rotation around an axis perpendicular to the two fields of view at a rate of $1 \% / \mathrm{min}$. Since the basic angle is $106.5^{\circ}$, the stars pass on the second field of view 106.5 minutes after the first one. The speed is optimized to put an upper limit on the across-scan smearing of stellar images (Lindegren \& Bastian 2011). In addition, the thermal stability requirements for the payload implies that the solar aspect angle is fixed (about $45^{\circ}$ ) i.e. the spin axis precesses around the solar direction. Gaia will scan the sky spinning around its axis with a period of about six hours. Due to its precession motion with a period of 63 days, Gaia will observe the entire sky in 183 days. Over the course of its five year mission, each source will therefore be observed on the $\mathrm{AF}$ and $\mathrm{BP} / \mathrm{RP}$ fields a number of times between about 40 and 250 times, depending primarily on its ecliptic latitude. To ease instrument testing and calibration, during the commissioning phase and early mission, a special scanning law (EPSL) is adopted, allowing multiple observations of the Ecliptic Poles. In that case, the direction of scanning changes by the same rate as the Sun (and spin axis) and moves along the ecliptic about $1^{\circ}$ per day. The main advantage of this configuration is that a relatively small set of objects near the ecliptic poles are observed many times in a short period (essentially twice per spin period). However, this scanning law is quite unfavorable to derive an astrometric solution. In the regions near the ecliptic, the scans are always parallel to each other, i.e. perpendicular to the ecliptic. This is fine for the determination of ecliptic latitude but not at all for the longitude, resulting in a degeneration when deriving the astrometric solution. In addition, long periods of EPSL can produce a substantial loss of uniformity in the coverage of the sky during the mission eventually degrading of the astrometric performances. For these reasons, the period of EPSL will be limited to a few weeks, as a compromise between the request of calibrating the instrument response and the need of reaching the targeted astrometric accuracy. 


\section{Gaia data processing}

\subsection{The astrometric solution}

The multiple observation epochs, taken from different points on Gaia's orbit around the Sun, are the basis for the astrometric analysis. A detailed description of the principles and methods to derive the astrometric solution for Gaia can be found in Lindegren et al. 2012. Gaia observations are made in the scanning reference system centered on the Gaia center of mass and rigidly connected to the body of the Gaia spacecraft. These observations need to be transformed in the International Celestial Reference System (ICRS), whose origin is located at the barycentre of the solar system and is fixed with respect to distant quasars Feissel \& Mignard 1998.

The astrometric solution for Gaia relies on the simultaneous determination of a very large number of unknowns for a small sample of $10^{8}$ primary sources, which are astrometrically well-behaved (mostly single stars and quasars).

The unknowns include:

1) the astrometric parameters for the primary sources which are related to the astrometric reference frame. This results in about $5 \times 10^{8}$ unknowns;

2) the attitude, giving the instantaneous orientation of the Gaia instrument in the celestial reference frame as a function of time $\left(4 \times 10^{7}\right.$ unknown);

3 ) the geometric instrument calibration, representing the mapping from pixels on the CCD detectors to angular directions ( $10^{6}$ unknown).

Finally the astrometric solution needs to be aligned with the ICRS. Each source is observed relatively to a large number of other sources simultaneously in the same field of view or in the complementary one, linked together by the attitude and calibration models. This makes the problem very complex, and a direct solution unfeasible. An iterative least-squares solution, referred to as the Astrometric Global Iterative Solution (AGIS) is derived, updating the unknowns in a cyclic sequence until convergence. Then, the resulting celestial reference frame, attitude and instrument calibration are applied to the processing of all observations. At least eighteen months of data are necessary to converge to an astrometric solution.

\subsection{Photometry}

The Gaia photometry has the primary goal of allowing the on-ground corrections of image centroids measured in the main astrometric field for systematic chromatic shifts caused by aberrations. In addition these low resolution spectra can be used to derive the classification of the Gaia objects, i.e. derive the astrophysical parameters (see below). As discussed in the previous sections, Gaia will provide photometric information as G band and BP/RP spectra and magnitudes. Detailed information about the pass-bands, the transformation relations, the stellar tracks and isochrones in the Gaia system can be found in (Jordi et al. 2010). On the Gaia BP/RP plane only small windows of variable size, depending on the magnitudes of the stars, are observed and transmitted to Earth. During the read-out process, the information from most windows is binned in the across scan direction of transit over 12 pixels. The main effect of this binning is a significant gain in readout noise, and in the telemetry, reducing the size of the generated data. The full $2 \mathrm{D}$ image resolution is maintained only in special cases, i.e. for selected calibration observations and for bright stars. BP/RP spectra have a length of about 40 pixels in the scanning direction. However, windows of 60 pixels are extracted and transmitted to Earth to sample image wings, and to allow background estimation. BP/RP spectra have low resolution $\mathrm{R}_{\mathrm{P}}=\lambda / \Delta \lambda=85-13$ in the $\mathrm{BP}$ and $26-17$ in the RP. Finally, to avoid 
saturation of bright stars, a gating system depending on the magnitude is activated, having the effect of reducing the exposure time.

\subsubsection{Spectra pre-processing}

The pre-processing operates on the raw dispersed images with the aim of extracting clean BP/RP spectra. The basic process will apply the basic CCD calibrations (gain and bias), subtract the sky background and remove the flux of close sources. The high accuracy of Gaia requires that the main characteristics of the systems are well known and well calibrated. Several effects require special attention, namely sensitivity variations across a CCD, saturation and in general deviations from linearity, electronic bias variations, and the Charge Transfer Inefficiency (CTI). In addition, the fact that only the pixels inside the windows are read, binned and transmitted to Earth further complicates the problem. To calibrate these effects, a detailed model of the reading process is necessary. This is obtained by means of pre-scan samples and virtual objects (predefined empty windows placed on the focal plane). The CTI is particularly critical for Gaia: since the pixels are read in TDI mode, the charge transfer during reading takes place at a speed very close to the image motion. It is of fundamental importance that the charge transfer works with the same efficiency for faint and bright stars and all over the mission. However, the solar wind high-energy protons in L2 will create new energy levels in the Si structure of the CCDs (charge traps) which can capture and after some time, release the electrons. These traps increase the CTI of the detector, eventually inducing large charge loss and distorting the stellar images. This can result in a bias that depends in a non-linear way on the brightness and several observational circumstances. In absence of any mitigation and calibration, this effect can degrade the end-of-mission astrometric accuracy from $\mu$ as to mas levels. To mitigate the CTI effect, periodic injection of charge into the lines of the CCD are planned to keep traps filled. The residual effects are calibrated using a model that can replicate the distortion of a sampled image due to radiation-induced trapping effects. Finally, this model is applied using a forward modeling technique in the pipeline processing (see Short, et al. 2013, Seabroke et al. 2013, Holl et al. 2012 and reference therein for a detailed description of the problem and of the methods).

\subsubsection{The photometric calibration}

The spectra calibration process involves two aspects: the internal and external calibration. The internal calibration model uses a few millions of well-behaved stars (internal standards), observed by Gaia to all observations to a reference instrument, on the same instrumental relative flux and wavelength scales. Several instrumental effects produce variations in the observational flux of the same source, i.e the field coating, the dispersion law, the CCD response. In addition, non-linearity effects are expected in case of very bright and very faint stars. Other effects such as full well capacity variations, background non- uniformity, contamination, CTI are all functions of the field of view and focal plane position (see Jordi et al. 2007, Fabricius et al. 2013, Busso et al. 2012 for a wider discussion).

The absolute (or external) calibration reports the relative flux scale of the internally calibrated spectra to an absolute flux scale in physical units and will be tied to the calibration of Vega within a few percent. The calibration set consists of a sample of 200-300 stars in the magnitude range $9<V<15$. The requirement is to reach an internal precision of $1 \%$ for the standards and a final accuracy on the external calibration of the order of 1-3\% (Cacciari 2011). These standards have been monitored for short term $(1-2 h)$ and long term $(3 \mathrm{yr})$ photometric variability to reject objects showing a intrinsic 
variations larger than the limit of $5 \mathrm{mmag}$, which is necessary to ensure the requested photometric accuracy and precision (Pancino et al. 2012).

\section{3. $R V S$}

RVS spectra are binned in the across-scan direction over 10 pixels, except for bright stars, for which single-pixel-resolution windows are used. Starting from an initial estimate of the source parameters obtained from astrometric and photometric information, the radial velocities are derived by cross-correlating spectra with a template (Guerrier et al. 2007) using an iterative procedure. Radial velocity determination is a function of the signal to noise ratio: for stars brighter than $15^{\text {th }}$ magnitude, it will be possible to derive radial velocities from single transit spectra while in the case of fainter stars, end-of-mission integrated spectra are necessary to derive of mission-averaged radial velocities.

\section{Gaia Accuracy}

The astrometric accuracy is a function mostly of SNR and ultimately of the G magnitude. The end-of-mission sky averaged parallax accuracy depends on the color of the stars (slightly better for earlier type stars) and is 25-26 $\mu \mathrm{as}, 137-145 \mu \mathrm{as}$, and $328-347 \mu \mathrm{as}$ at $G=15,18.5$, and 20 , respectively. The proper motion accuracies in $\mu$ as $/ y r$ are about 0.5 times the size of the parallax accuracies. The performances of the bright stars having $5.4<G<14$ depend on the gating scheme and are of the order of $7-17 \mu$ as. The 6000 brightest stars in the Gaia sky, having $G<5.5$, will be saturated. In principle the performance on parallax and position errors scale with mission length $\mathrm{L}$, as $L^{0.5}$, and as $L^{1.5}$ for the proper motions. However, the real performances might be quite different, since early in the mission the astrometric solution might be affected by systematic errors. At the end of the mission, the parallaxes and proper motions will have an accuracy better than $10 \%$ for a main sequence star of spectral type $\mathrm{G}$, with $\mathrm{M}_{\mathrm{V}}=4.5$, within $2.5-3 \mathrm{kpc}$. The solar vicinity, defined as the distance where a complete sample is available at $10 \%$ accuracy, will go from the $50 \mathrm{pc}$ of Hipparcos to $2.5-3 \mathrm{kpc}$ for un-evolved main sequence star brighter than $\mathrm{M}_{\mathrm{V}}=4.5$ (Vallenari et al. 1999). For bright halo stars (giants, RRLyrae), Gaia will give a complete sample at $10 \%$ accuracy within $10 \mathrm{kpc}$, including the inner halo in the solar vicinity.

The sources' G-band magnitudes are measured with very high SNR: and the accuracy will be limited by the calibration error. The end-of-mission accuracy on $G$ magnitude is $1-3 \mathrm{mmag}$, even at $G=20$. Performances will be slightly worse for the $\mathrm{G}_{\mathrm{BP}}, \mathrm{G}_{\mathrm{RP}}$ magnitudes, being limited by calibration noise for bright magnitudes and by photon noise at the faint end. For $\mathrm{G}_{\mathrm{BP}}, \mathrm{G}_{\mathrm{RP}}$ the photometric errors are ranging from $4 \mathrm{mmag}$ at $\mathrm{G}<15$ to $43 \mathrm{mmag}$ at $\mathrm{G}=20$.

The radial velocity precision ranges from $1-15 \mathrm{~km} \mathrm{~s}^{-1}$, as a function of the color and magnitude (de Bruijne 2012). This precision translates in the end-of mission precision on the transverse velocity as described in Fig. 2.

\section{Gaia Classification}

To avoid biases, Gaia will re-classify the observed objects, deriving their astrophysical parameters (AP). This classification will be part of the Gaia catalog released to the scientific community, but will also define the input parameters for the RVS, the variability analysis and will identify the QSOs to build the reference frame. The classification algorithms are based on both supervised and unsupervised methods, first producing a 
discrete classification of the objects, i.e. dividing objects having higher probability of being stars, galaxies, and QSOs, then deriving the astrophysical parameters for each class of objects (stars, galaxies, QSOs). A detailed description of methods and performances can be found in Bailer-Jones et al. (2013).

Supervised methods require the comparison with a set of templates, either observational or synthetic, as training data sets. Large sets of synthetic libraries of spectra of stars, galaxies, and QSOs have been calculated by the Gaia community (see Sordo et al. 2011 for a comprehensive description). Stellar libraries are produced using codes optimized for a given $\mathrm{T}_{\text {eff }}$ range, or for a specific object type, including when appropriate: non-local thermodynamical equilibrium; dust; mass loss; circumstellar envelopes; magnetic fields; stellar rotations; variations of single element abundances; chemical peculiarities. Clearly, training data cannot be purely synthetic, since current models have difficulties to satisfactorily simulate some process such as emission line objects, or cool stars. To cover these specific cases and to build a set of reference stars, observational programs are ongoing (Soubiran et al. 2008).

Large synthetic libraries of galaxy spectra as training data, covering the main Hubble types in the Gaia spectral range have been calculated using the code PEGASE.2 (Fioc \& Rocca Volmerange 1997). Four spectral types have been simulated: early, spiral, irregular, quenched star formation. Each galaxy type is characterized by four main parameters: the timescale of in-falling gas and three parameters which define the appropriate star formation law. The current library has been optimized through comparisons with a sample of SDSS galaxies. It includes 28,885 synthetic galaxy spectra simulated at a range of redshifts from 0 to 0.2 , and a range of values of $\mathrm{A} 0$ from 0 to $10 \mathrm{mag}$ in order to simulate extinction due to the interstellar medium of our Galaxy (Tsalmantza et al. 2009, Karampelas et al. 2012). In addition a semi-empirical library of 33,670 galaxy spectra has been produced fitting SDSS spectra to the synthetic galaxy library (Tsalmantza et al. 2012).

Since the objects are classified by different software modules using different methods any given source may be assigned multiple sets of APs, depending on the data used (RVS,

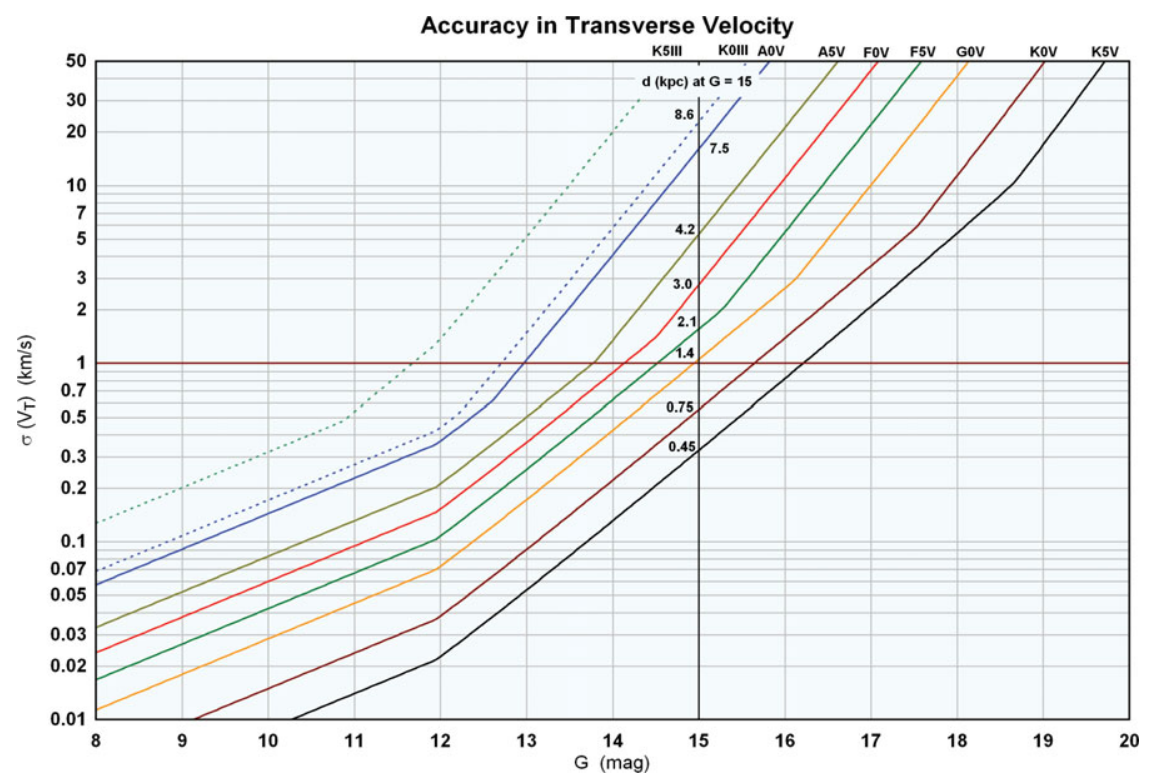

Figure 2. Transverse velocity precision as function of the G magnitude, of the spectral type and of the distance. Image courtesy of F. Mignard 
Table 1. Performances expected from parameter determination Bayesian algorithms. For a full discussion, see Bailer-Jones et al. 2013.

\begin{tabular}{ccccc}
\hline$G$ & stellar type & $\mathrm{T}_{\text {eff }}$ & $\log g$ & {$[\mathbf{F e} / \mathbf{H}]$} \\
\hline \multirow{2}{*}{15} & $\mathrm{~A}$ & 260 & 0.38 & 0.9 \\
& $\mathrm{G}$ & 140 & 0.22 & 0.16 \\
& $\mathrm{M}$ & 70 & 0.29 & 0.25 \\
\hline \multirow{2}{*}{19} & $\mathrm{~A}$ & 400 & 0.51 & 0.74 \\
& $\mathrm{G}$ & 450 & 0.45 & 0.65 \\
& $\mathrm{M}$ & 90 & 0.17 & 0.29 \\
\hline
\end{tabular}

$\mathrm{BP} / \mathrm{RP}$, parallaxes, proper motions), but also on the training data libraries. All of these sets of APs will be reported in the data releases, providing however the best set of APs for each source.

\subsection{Stars}

Single stars are the main Gaia targets. The goal of the Gaia classification is to provide $\mathrm{T}_{\text {eff }},[\mathrm{Fe} / \mathrm{H}], \log g$ and the line-of-sight extinction, $\mathrm{A}_{0}$. To check the internal consistency of the results, multiple algorithms will be used to derive their parameters using $\mathrm{BP} / \mathrm{RP}$ spectra. These include $i$ ) Support Vector Machine (SVM) method, ii) a Bayesian method employing a forward model and a Monte Carlo algorithm to sample the posterior probability density function over the APs; iii) an iterative Newton-Raphson minimization algorithm used to find the best fitting forward-modeled spectrum, its APs and their covariances. Each of these algorithms give better performances in different parts of the AP space. For instance using the Bayesian method, the accuracies are shown in Table 1.

For the stars having RVS spectra, the performance estimates (the $70 \%$ quartile of the error distribution) for simulated FGK type disk stars are around $100 \mathrm{~K}$ in $\mathrm{T}_{\text {eff }}, 0.15$ dex in $\log g$, and $0.11 \mathrm{dex}$ in $[\mathrm{Fe} / \mathrm{H}]$ at $\mathrm{G}_{\mathrm{RVS}}=12$; and $300 \mathrm{~K}$ in $\mathrm{T}_{\text {eff }}, 0.40$ dex in $\log g$ and 0.3 dex in $[\mathrm{Fe} / \mathrm{H}]$ at $\mathrm{G}_{\mathrm{RVS}}=14.5$ (Kordopatis et al. 2011). The individual abundances of several elements (Fe, Ca, Ti, Si) will be measured for brighter stars having $\mathrm{G}_{\mathrm{RVS}}<13$, with an internal precision of 0.1 dex.

\section{2. $Q S O s$}

Gaia is expected to observe about 500,000 QSOs. The BP/RP spectra are analyzed by a SVM algorithm to identify rare objects, such as QSOs. About 70\% of the expected QSOs are expected to be correctly identified. Then, they are classified into the three classes: type I, type II and BAL (Broad Absorption Line quasars). Finally, the red-shift, total emission line equivalent width, and the slope of the power law continuum are estimated using SVM and Extremely Randomized Tree algorithm. The SVM classifier achieves an accuracy of $97.4 \%, 95.8 \%$, and $91 \%$ for $G=15,18.5$, and 20 (respectively). The red-shift (in the range $0-5.6$ ) is derived with an accuracy of $0.02,0.03$, and 0.04 for $G=15,18.5$, and 20 respectively.

\subsection{Gaia point-like galaxies}

The high resolution Gaia observations will allow the detection of small galaxies that could not be resolved from ground base surveys such as the Sloan Digital Sky Survey, giving us a unique chance to access a whole sky survey of these objects, something that no ground-based survey has ever recorded. Gaia will observe a few million unresolved galaxies with point-like cores at the diffraction limit. They are expected to be in the local Universe, having a red-shift $\mathrm{z}<0.4$, with only a minority in the range $0.2<z<0.4$. Gaia galaxy classification is focused on these point-like galaxies, and it is aimed to identify the 
galaxy type, and estimate the red-shift, the total Galactic extinction towards the source, and the star formation law in the source galaxy using an SVM algorithm. The galaxy type classification performance, defined as the percentage of true-positive classifications is $98 \%, 89 \%$, and $71 \%$ for $G=15,18.5,20$, respectively. However, the performances are higher for the spirals and quenched galaxies $(>98 \%)$ and the lowest for the irregular types $(89 \%)$. The red-shift can be estimated up to 0.2 with an uncertainty of 0.002 , and 0.028 going from $G=15$ to $\mathrm{G}=20$. Finally, the Galactic extinction (in the range $0-6 \mathrm{mag}$ ) is derived with an uncertainty of 0.04 to 0.35 for $G=15,20$ respectively.

\section{Gaia special objects}

\subsection{Crowded regions}

Gaia is limited by two effects. First, the dust extinction introduces a degeneracy between extinction and effective temperature determinations on the basis of the BP/RP data, mainly at faint magnitudes. High extinction regions will not be visible to Gaia. Second, Gaia is confusion limited (images of different stars are superposed on the FoV). The astrometric instrument can handle very high densities of the order of 1,000,000 objects $\mathrm{deg}^{-2}$. The $\mathrm{BP} / \mathrm{RP}$ is confusion limited when the total star density per transit (sum

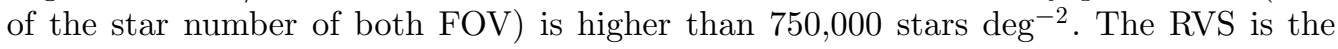
instrument facing the more severe limitations, due to the object density: RVS can handle densities up to 36,000 objects $\mathrm{deg}^{-2}$. This means that the central inner degrees of the Galaxy bulge and disk will not be well measured (Wilkinson et al. 2005).

\subsection{Globular clusters}

The crowding of stellar images is quite large in globular clusters. For this reason, it is expected that Gaia performances are very poor inside the half mass radius. However, Gaia contribution to the globular cluster science can be significant in the external part of the clusters. 80 out of 150 clusters are at distances smaller than $10 \mathrm{kpc}$ (Bica et al. 2006). The magnitudes of the horizontal branch will range from $V=12.5$ for the closest to $V=19$ for clusters in the Large Magellanic Cloud: all the known globulars in the Galaxy and in the Magellanic Clouds will be observed down to the horizontal branch. However, due to the limiting magnitude of Gaia, only clusters located closer than $15 \mathrm{kpc}$ can be sampled down to the turnoff (Pancino et al. 2013). The superb quality of the proper motions will allow to separate bright cluster stars from field contaminants even in crowded regions such as the Galactic center direction. In practice, 100 to 10000 stars per cluster will be measured by Gaia. This will allow the determination of statistical distances with accuracy of a few percent for all the known Galactic globular clusters.

\subsection{Open clusters}

Gaia will bring us in a new domain of open cluster (OC) research. Gaia will measure distances for individual stars in OCs with a precision better than $1 \%$ for clusters closer than $1 \mathrm{kpc}$ and better than 10\% for almost the entire OC family. Higher accuracies are expected for proper motions, yielding a precision in individual tangential velocities of the order $0.2-0.3 \mathrm{~km} \mathrm{~s}^{-1}$ for low mass stars in clusters up to $1.5 \mathrm{kpc}$, and up to larger distances for bright $\mathrm{O} / \mathrm{B}$ stars. This would allow resolving both peculiar velocities and internal dispersions, which are typically of the order of $0.8-3 \mathrm{~km} \mathrm{~s}^{-1}$ (Furesz et al. 2008). Gaia will also provide good photometric information, helping to characterize cluster members. Fig. 3 presents the simulated colour magnitude diagram of one of the most distant OCs Be 29, located at $13.05 \mathrm{Kpc}$ from us, having an age of 3.7 Gyrs (Bragaglia \& Tosi 2006) when the photometric errors in the Gaia passbands are included. 


\subsection{Extended objects}

Gaia has a limited capability to detect some extended objects. Since the transit angle changes from one observation to another, it is possible to numerically stack on ground these one-dimensional windows to produce a two dimensional map of their surrounding inside a radius $<3^{\prime \prime}$. However, not all of the extended objects can be sent to ground, since the on-board selection algorithm is tailored to select star-like profile. Therefore many extended objects, such as some solar system objects and extended galaxies will be filtered out. Simulations have shown that the vast majority of the elliptical galaxies (pure bulge) will be sent to ground while lower detection probability is found for disky galaxies (having disc radius of the order of 2.0") (Krone-Martins et al. 2013).

\section{Data release scenario}

At present five data releases are foreseen. However, these plans assume smooth operations and might be subject to changes due to unexpected conditions (Prusti 2012, Brown 2012).

- The first release (launch +22 months) will contain positions and the mean $G$ magnitudes of single non-variable sources, and their errors. In addition, a catalog of proper motions (HTPMC) will combine astrometric data from the Hipparcos and Gaia missions on a 23-year baseline, and produce proper motions for all Hipparcos stars. It should be noticed that the proper motions will be absolute, so avoiding the possible regional systematic distortions of the order of $10,000 \mu \mathrm{as} / \mathrm{yr}$ or more present in modern, massive, ground-based catalogs like UCAC-3, which generally have only relative proper motions. In the HTPMC, the expected accuracy on the proper motions will be 30 to $250 \mu \mathrm{as} / \mathrm{yr}$, depending on the magnitude, with an average of $50 \mu \mathrm{as} / \mathrm{yr}$ (20 times better than the average $1000 \mu \mathrm{as} / \mathrm{yr}$ of the Hipparcos values). These data can be coupled with spectroscopic and photometric literature data. A recent compilation available for Hipparcos stars is given by Anderson \& Francis (2012), where 46,392 radial velocities, 18,549 homogenized iron abundances $[\mathrm{Fe} / \mathrm{H}]$ are taken from the literature, together with 2MASS photometry. In addition, the Gaia-ESO Survey will deliver metal abundances and radial velocities from high resolution spectroscopy for a sample of 5000 field stars and for several clusters in the solar vicinity. Complemented by these data, the HTPMC will be of fundamental importance to study the properties of the nearby stars. This Catalog will be the reference for the solar vicinity in the coming years, since the precision on proper motions of the HTPMC will be superseded only with the final Gaia Catalog release.

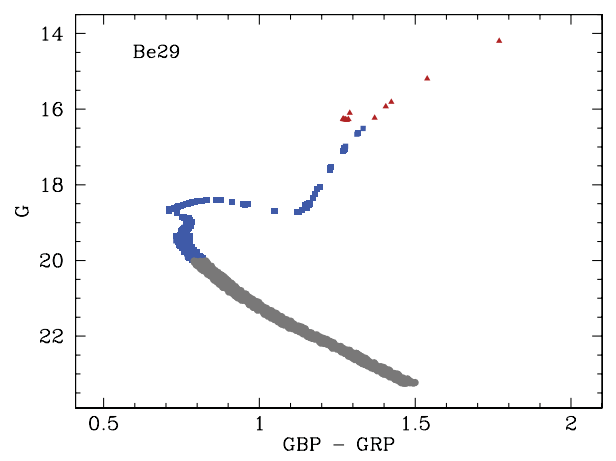

Figure 3. Simulated colour-magnitude diagram of Be 29 as seen by Gaia. Red triangles indicate the objects having radial velocity measurements and blue squares show the stars for which the astrometry is available. 
- The second release is planned at launch +28 months. This release will contain the five parameters of the astrometric solution for each source, i.e., the position, proper motion and parallax, together with their errors; the mean $G$ magnitude and the first results of the mean integrated $\mathrm{BP} / \mathrm{RP}$ spectro-photometry, and radial velocities for bright stars showing non-variable behavior. A preliminary classification of the Gaia objects based on these data will be provided.

- The third release (launch +40 months) will contain an update of all the quantities published in the previous releases; orbital solutions for short period variables (periods between 2 months and $75 \%$ of the observation duration); mean spectro-photometry $B P$, $R P$, RVS products and astrophysical parameters of the stars based on their $\mathrm{BP} / \mathrm{RP}$ spectra.

- The fourth release (launch + 65 months) will deliver an update of the above quantities and will include variable stars, non-single stars, and solar system objects

- The fifth release (end-of-mission operation +3 years) will contain all the data products that have been defined: astrometry, photometry, spectra, radial velocities,non- single stars, list of exoplanet candidates, solar system objects, the variable star catalog and astrophysical parameters.

\section{References}

Anderson \& Francis 2012, AstL 38, 331

Bailer-Jones, C. A.L., Andrae, R., Arcay,B., Astraatmadja, T., Bellas-Velidis, J., Berihuete, A., Bijaoui, A., et al. 2013, A\&BA submitted

Bica, E., Bonatto, C., Barbuy, B., \& Ortolani, S., 2006, A\& $A, 450,105$

Bragaglia, A., Tosi, M., 2006, AJ, 131, 1544

Brown, A., 2012, Proceedings of the meeting Dynamics Meets Kinematic Tracers, Online at http://mpia.de/ dynamics/ringberg/?view=program, id.37

Busso, Giorgia; De Angeli, Francesca; Montegriffo, Paolo, 2012, SPIE, 8442, 42

Cacciari, C., 2011, EAS Publications Series, Volume 45, 2011, pp. 155-160

de Bruijne,J. H.S, Kohley, Prusti, T. 2010 in Oschmann J. M., Jr., Clampin M. C., MacEwen H. A., eds, SPIE Conf. Ser Vol. 7731

de Bruijne,J. H.S, 2012, Ap\&SSS, 341, 31

Dopita, M. A., Marconi, M., Clementini, G., Brocato, E,, 2012, Ap\&SSS, 341,1

Drew, J. E., 2012, sngi.conf, 15

Fabricius, C., Jordi, C., Carrasco, J. M., Voss, H., Weiler, M. Proceedings of the X Scientific Meeting of the Spanish Astronomical Society, 2013, Eds.: J. C. Guirado, L. M. Lara, V. Quilis, and J. Gorgas., pp.880-885

Feissel, M., Mignard, F., 1998, A\&A 331, L33

Fioc, M. \& Rocca-Volmerange, B. 1997, A\&A, 326, 950

Freeman, K. C 2012, in Galactic Archaeology, eds by W. Aoki, M. Ishigaki, T. Suda, T. Tsujimoto, and N. Arimoto, ASP Conference Proceedings, Vol. 458, p 393

Furesz, G., Hartmann, L. W., Megeath, S. Thomas; Szentgyorgyi, A. H., Hamden, E. T., 2008, ApJ 676, 1106

Gilmore, G., Randich, S., Asplund, M., Binney, J., Bonifacio, P., Drew, J., Feltzing, S., Ferguson, A., Jeffries, R., Micela, G., and 23 coauthors, 2012 Msngr., 147, 25

Guerrier, A., Katz, D., Turon, C., Crifo, F. SF2A-2007, Proceedings of the Annual meeting of the French Society of Astronomy and Astrophysics held in Grenoble, France, July 2-6, 2007, Eds.: J. Bouvier, A. Chalabaev, and C. Charbonnel, p.485

Holl, B., Prod'homme, T., Lindegren, L., Brown, A. G. A.,2012, MNRAS, 422, 2786

Karampelas, A., Kontizas, M., Rocca-Volmerange, B., et al. 2012, A\&AA, 538, A38

Kordopatis, G., Recio-Blanco, A., de Laverny, P., et al. 2011, A\&A, 535, A106

Krone-Martins, A., Ducourant, C., Teixeira, R., Galluccio, L., Gavras, P., dos Anjos, S., de Souza, R. E., Machado, R. E. G., Le Campion, J.-F., 2013, arXiv1307.5732K 
Jordi, C., Carrasco, J. M., 2007, ASP Conference Series, Vol. 364, Edited by C. Sterken. San Francisco: Astronomical Society of the Pacific, p.215

Lindegren, L., Bastian, U, 2011, EAS Publications Series, Volume 45, 2011, pp.109-114

Lindegren, L., Lammers, U., Hobbs, D., O'Mullane, W., Bastian, U., Hernndez, J., 2012,

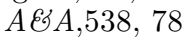

Majewski, S.R, 2010, in Stellar Populations Planning for the Next Decade, Proceedings of IAU Symposium, Volume 262, p. 99-110

Pancino, E., Altavilla, G., Marinoni, S., Cocozza, G., et al. 2012 MNRAS 4261767

Pancino, E., Bellazzini, M., \& Marinoni, S., 2013, MemSait v.84, p.83

Prusti, T. 2012, Astronomische Nachrichten, 333, 453

Saito, R. K., Hempel, M., Minniti, D., Lucas, P. W., Rejkuba, M., Toledo, I., Gonzalez, O. A., Alonso-Garca, J., Irwin, M. J., Gonzalez-Solares, E., \& 100 coauthors, 2012, A $\& A$, 537, $107 \mathrm{~S}$

Seabroke, G. M., Prod'homme, T., Murray, N. J., Crowley, C., Hopkinson, G., Brown, A. G. A., Kohley, R., \& Holland, A., 2013, MNRAS, 430, 3155

Siebert, A., in SF2A-2012: Proceedings of the Annual meeting of the French Society of Astronomy and Astrophysics. Eds.: S. Boissier, P. de Laverny, N. Nardetto, R. Samadi, D. Valls-Gabaud and H. Wozniak, pp.121-127

Short, A. C., Crowley, J. H. J., de Bruijne, \& T. Prodhomme, 2013 MNRAS, 430, 3078

Sordo, R., Vallenari, A., Tantalo, R., et al. 2011, Journal of Physics Conference Series, 328, 12006

Soubiran, C., Allende Prieto, C., Altmann, M., Bragaglia, A., Clementini, G., Frmat, Y., Heiter, U., Joliet, E., Pancino, E., Sartoretti, P., \& 2 coauthors, 2008, SF2A-2008, Proceedings of the Annual meeting of the French Society of Astronomy and Astrophysics Eds.: C. Charbonnel, F. Combes and R. Sama

Sozzetti A., 2013, in Hot Planets and Cool Stars, Garching, Germany, ed. Roberto Saglia; EPJ Web of Conferences, Volume 47, id.15005

Tanga, P. \& Mignard, F., P\&SSS, 73, 5

Turon, C., Meynadier, F. \& Arenou, F. (Eds) 2011, ELSA Conference 2010: Gaia at the Frontiers of Astrometry, EAS Pub. Series, Vol. 45

Turon, C., Luri, X., \& Masana, E., 2012, Ap\&SSS, 341, 15

Tsalmantza, P., Kontizas, M., Rocca-Volmerange, B., et al. 2009, A\&\&, 504, 1071

Tsalmantza, P., Karampelas, A., Kontizas, M., et al. 2012, A\&A, 537, A42

Vallenari A., Bertelli G, Bressan A., et al 1999, BaltA

Wilkinson, M. I., Vallenari, A., Turon, C., Munari, U., Katz, D., Bono, G., Cropper, M., Helmi, A., Robichon, N., Thevenin, F., Vidrih, S., Zwitter, T., Arenou, F., Baylac, M.-O., Bertelli, G., Bijaoui, A., Boschi, F., Castelli, F., Crifo, F., David, M., Gomboc, A., Gomez, A., Haywood, M., Jauregi, U., de Laverny, P., Lebreton, Y., Marrese, P., Marsh, T., Mignot, S., Morin, D., Pasetto, S., Perryman, M., Prsa, A., Recio-Blanco, A., Royer, F., Sellier, A., Siviero, A., Sordo, R., Soubiran, C., Tomasella, L., \& Viala, Y. 2005, MNRAS 359, 1306 